\title{
Sonido: Sonómetro analógico y composición de movimientos armónicos para la demostración en el aula
}

Prof. Romina Del Milagro Gilabert*, Ing. Cesar A. Cabrera*, Ing. Carlos A. Merino*, Dra. Erika N. Bentz* y Dr. Patricio F. Provasi*

\section{Resumen}

Las figuras de Lissajous permiten de forma gráfica analizar e interpretar la interacción sobre una partícula de dos movimientos armónicos simples perpendiculares entre sí, proceso que, de otra forma sería más complejo de comprender.

En este trabajo se presenta una propuesta didáctica con la intención de favorecer el proceso Enseñanza-Aprendizaje basada en el diseño de un dispositivo sencillo y de bajo costo para la demostración del fenómeno de superposición de movimientos armónicos simples en el aula de física. El mismo consiste básicamente en una membrana de látex o poliuretano, con un pequeño espejo en el centro montada sobre un bastidor. La membrana vibra de- bido al sonido que emerge de un parlante cercano que se controla con una App generadora de ondas. Un módulo Led-Láser produce un haz que se refleja en el espejo adherido a la membrana y proyecta ésta sobre una pantalla convenientemente situada a una distancia determinada. En la pantalla se observan las diferentes figuras de Lissajous que varían con la frecuencias y formas de onda generadas por la App. Midiendo la amplitud de las figuras formadas en la pantalla en función del volumen del parlante es posible determinar el valor de la intensidad de una onda sonora mono o poli-cromática.

\section{Palabras Claves}

Sonido. Intensidad. Frecuencia. Suma de frecuencias. Figuras de Lissajous

*Departamento de Física, FaCENA - UNNE - 0379-475-0698, patprovasi@gmail.com 


\section{Abstract}

The Lissajous' figures allow of a graphical form to analyze and to interpret the interaction on a particle of two simple harmonic movements perpendicular between themselves, process that, of another form would be more complex of unders tanding. In this work a didactic proposal appears with the intention of favoring the process Education - Learning based on the design of a simple device and of low cost for the demonstration of the phenomenon of superposition of simple harmonic movements in the physics' classroom.

The dispositive consists basically in a membrane of latex or polyurethane, with a small mirror of the center mounted on a frame. The membrane vibrates due to the sound that emerges of a nearby speaker which is controlled by an waves generator App. A module Led-Laser produces a beam that is reflected in the mirror adhered to the membrane and projects it on a screen suitably placed at a certain distance. On the screen is observed the different Lissajous' figures that change with the frequencies and waveforms generated by the App. Measuring the largeness of the figures formed on the screen according to the volume of the speaker it is possible to determine the value of the intensity of a mono- or poly-chromatic sonorous wave.

\section{Keywords}

Sound. Intensity. Frequency. Sum of frequencies. Lissajous’ Figures.

\section{Introducción}

Los experimentos de cátedra son un recurso docente ampliamente utilizado en las Ciencias Naturales para demostrar e ilustrar fenómenos físicos que se explican de modo teórico durante las clases. Suelen provocar la sorpresa del estudiante, atrayendo su atención y haciendo que el alumno se interrogue sobre los procesos que están teniendo lugar. Generan así un contexto propicio que facilita una mejor comprensión de los contenidos teóricos y de su aplicación práctica ya que: incrementan la atención de los estudiantes, promueven su curiosidad aumentando la motivación y conectan los conceptos explicados en clase con su vida cotidiana.

En la constante búsqueda del empleo de más y mejor estrategias que apoyen los procesos de Enseñanza Aprendizaje dentro y fuera del aula de clase; el dispositivo diseñado se caracteriza por su fácil y económica construcción, por su carácter visual y por la invalorable posibilidad que brinda a los estudiantes de interaccionar con él.

Las figuras de Lissajous resultan visualmente muy atractivas para el público además del hecho que nos permite analizar gráficamente la composición de movimientos armónicos simples, que de otra forma, sin la ayuda visual resulta de una complejidad más elevada.

Otra importante motivación en el presente trabajo es la posibilidad de medir el nivel de sonoridad producido por un parlante, o de otra forma un sonómetro.

Entre la enorme variedad de fenómenos 
ondulatorios que tienen lugar en la naturaleza hay dos con los que estamos familiarizados, ya que tenemos órganos específicos para su recepción; ellos son la luz y el sonido [1].

El sonido es sensación fisiológica que producen las ondas acústicas, es un fenómeno físico producido por las vibraciones de objetos materiales, es una onda mecánica y como tal para transmitirse necesita de un medio material elástico (sólido, líquido o gaseoso).

El sonido de la voz se debe a las vibraciones de las cuerdas vocales. Un nadador oye debajo del agua la conversación de personas o el ruido de un barco; es decir que los líquidos transmiten el sonido. Si se apoya el oído sobre las vías de un ferrocarril, puede escucharse la marcha de un tren a distancia.

El oído es el órgano de la audición. El pabellón auditivo capta las ondas sonoras y las analiza a través del conducto auditivo externo hacia el tímpano. Este vibra y produce las ondas mecánicas que son transmitidas por los huesecillos del oído medio. El caracol, canal enrollado en espiral y lleno de un líquido contiene el órgano de Corti. Las ondas transmitidas por el líquido en forma de onda de presión excitan las células ciliadas del órgano de Corti, que envían impulsos al cerebro por medio del nervio auditivo [2].

Se presenta aquí una propuesta didáctica en búsqueda de integrar el proceso Enseñanza-Aprendizaje basada en el diseño de un dispositivo sencillo y de bajo costo que puede usarse para la demostración del fenómeno de superposición de movimientos armónicos simples en el aula de física. Se pretende además que el mismo favorezca la integración de conocimientos por parte del alumno al facilitar su complementariedad a otras herramientas y disciplinas de las Ciencias Naturales. Como ejemplo podemos mencionar algún software para programación y así poder realizar la simulación de este fenómeno, o bien alguna planilla de cálculo con el propósito de practicar el descubrimiento de alguna "ley natural" como se pretende mostrar en este trabajo al relacionar la sonoridad con la amplitud de las figuras de Lissajous formadas en la pantalla.

\section{Intensidad del sonido}

$\mathrm{El}$ ruido normalmente es un sonido que produce una sensación desagradable, que es el caso de una onda provocada por vibraciones extremadamente irregulares, pero la clave está en su intensidad [3].

La intensidad (I) de una onda es la potencia $(\mathrm{P})$ por la unidad de superficie $(\mathrm{S})$ que llega al sistema receptor.

Las ondas mecánicas en una cuerda son bidimensionales y se propagan en una dirección perpendicular al del movimiento ondulatorio. Las ondas sonoras, sin embargo, son tridimensionales. Un foco sonoro emite ondas esféricas que se propagan radialmente en el espacio. Esto tiene una consecuencia inmediata sobre la intensidad. Si el medio no atenúa la energía de la onda, la intensidad de ésta debe variar con la distancia (r) al cuadrado con relación al foco sonoro, pues por definición:

$$
I=\frac{P}{s}=\frac{P}{4 . \pi r^{2}}
$$


El umbral de audición es la intensidad mínima captada por el oído humano. Depende de la frecuencia del sonido. Así para una frecuencia de $1000 \mathrm{~Hz}$ el umbral equivale a $I_{0}=10^{-12} \frac{\mathrm{wall}}{\mathrm{m}^{2}}$

E1 sonido producido en un concierto de rock, o por un avión al despegar posee una intensidad de $10^{-1} \frac{\text { walt }}{m^{2}}$ y $100 \frac{\text { watt }}{m^{2}}$ respectivamente. En el primer caso está muy próximo al umbral del dolor y en el segundo es la intensidad a partir de la cual se daña el oído en forma permanente.

\section{El sonómetro y la escala decibélica}

Recibe el nombre de sonómetro el instrumento empleado para medir niveles de presión sonora. La unidad con la que trabaja $j$ es el decibelio. Los elementos principales de un sonómetro son: el micrófono, el preamplificador, el amplificador y un dispositivo de lectura analógica o digital. Dicho instrumento procesa los niveles de ruido a medida que los recibe y los muestra sucesivamente $o$ a intervalos de tiempo determinados. Algunos sonómetros proporcionan medidas integradas, puesto que facilitan también datos de niveles de ruido que se han rebasado durante un porcentaje determinado del tiempo de medición o bien en un intervalo determinado de frecuencias [4].

Habitualmente los sonómetros se clasifican, en función de su precisión decreciente en decibelios (dB), en tres clases. Los sonómetros de clase 0 se usan estrictamente en laboratorios, los de clase 1 en entornos urbanos y los de clase 2 en entornos laborales.
Se define nivel de intensidad del sonido como:

$$
\beta=10 . \log \left(\frac{I}{I_{0}}\right)
$$

Esta es una magnitud sin unidades, pero, de todas formas, se la vincula a la unidad $d b$, en memoria a Alexander Graham Bell (1847-1922).

\section{Figuras de Lissajous}

Jules Antoine Lissajous (1822-1880) fue un físico francés. En 1855 ideó un método óptico simple para el estudio de vibraciones compuestas, colocando un pequeño espejo a cada uno de los objetos que vibran (dos diapasones, por ejemplo) y apuntaba un haz de luz a uno de los espejos. E1 haz se refleja en primer lugar al otro espejo y de allí a una gran pantalla, donde se formaba un patrón bidimensional como resultado visual de la combinación de las dos vibraciones. Esta sencilla idea es el precursor del moderno osciloscopio, que fue una novedad en la época de Lissajous, hasta entonces el estudio de sonido dependía únicamente del proceso de audición; es decir, solo por el oído humano. Lissajous literalmente hizo posible "ver el sonido" $[4,5,6]$.

Una descripción técnica de las figuras de Lissajous (Figura 1) puede resumirse como los patrones formados al superponer dos representaciones de vibraciones periódicas, a lo largo de dos ejes perpendiculares. La definición paramétrica de las figuras de Lissajous está dada por la siguiente ecuación: 


$$
x=a \operatorname{sen}(\omega 1 t+\varphi 1) \text { e } y=b \operatorname{sen}\left(\omega_{1} t+\varphi_{1}\right)
$$

En las ecuaciones (3) $\omega 1$ y $\omega 2$ son las frecuencia angular (en radianes por segundo), $a$ y $b$ son las amplitudes respectivas, $\varphi 1$ y $\varphi 2$ son las diferencias de fase entre una función y la otra y $t$ el parámetro de tiempo.

Dependiendo de las condiciones iniciales de este sistema se pueden obtener distintas formas, como las representada en la Fig. 1, habría que manipular seis parámetros diferentes, lo que hace complejo el control de este sistema.

Figura 1: Ejemplo de figuras típicas de Lissajous para varias relaciones de frecuencia vertical:horizontal [1:1, 1:2, 1:3, 3:4, 5:6] y varias relaciones entre los ángulos de fase $[0, \pi / 4, \pi / 2,3 \pi / 4, \pi]$.

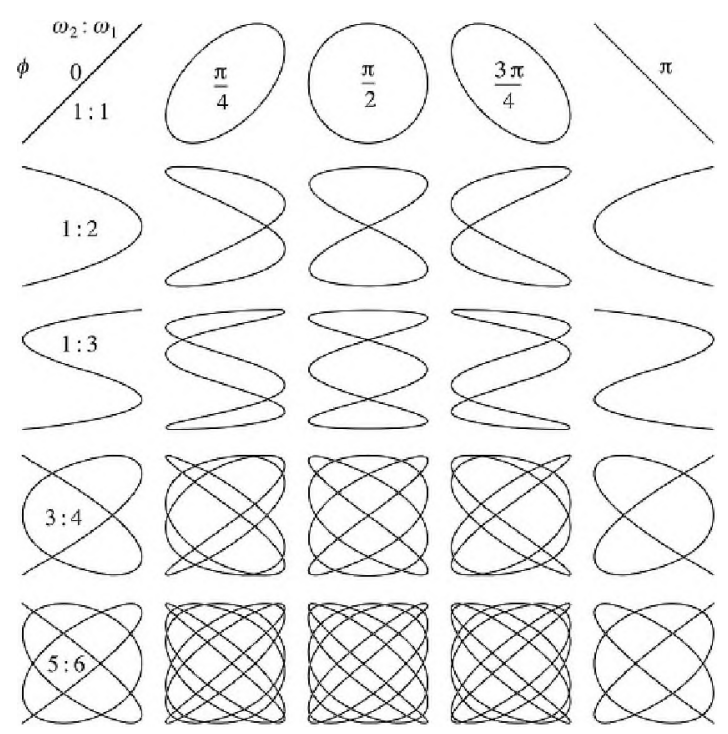

\section{Materiales y Métodos}

\section{Elementos a utilizar}

- App generadora de frecuencias de sonido (Ej. Frequency Sound Generator V2.27 Fine Chromatic Tuner, Mannheim Germany).

- Sonómetro (Ej. Sound Meter-Decibel V1.2.0 - Melon Soft).

- Parlante con conexión a teléfono celular.

- Soporte con membrana de poliuretano y una placa que refleje la luz (puede ser un trozo de espejo liviano).

- Un Led-Láser y un soporte.

- Una pantalla (puede ser una cartulina blanca).

- Cinta métrica.

- Regla de $30 \mathrm{~cm}$.

Figura 2: Esquema del dispositivo sonómetro/membrana vibrante donde se representan sus partes principales.

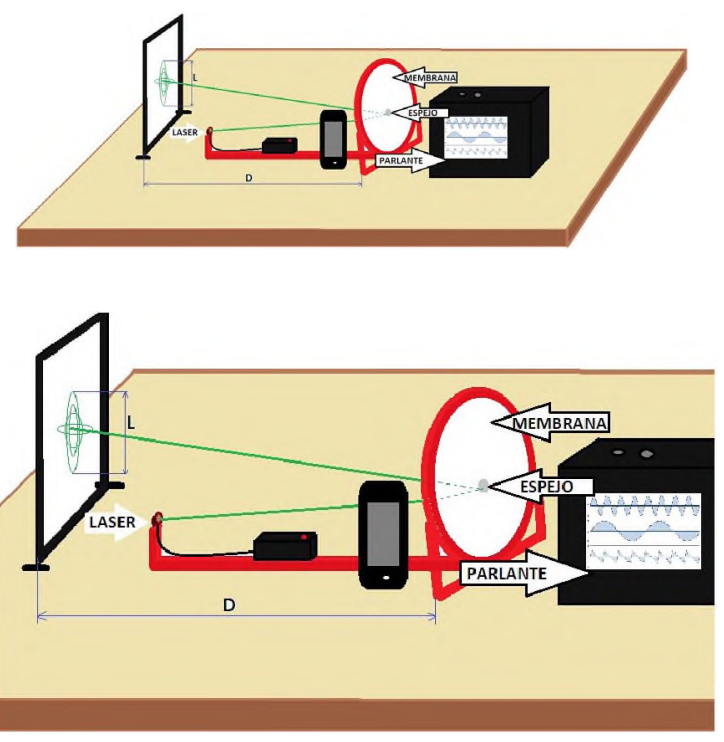


Para realizar el experimento se debe colocar en un extremo de una superficie firme el dispositivo que sostiene el láser y la membrana junto con el parlante, en el otro extremo colocar la pantalla donde se proyectara la imagen (tratar que la separación $\mathrm{D}$ entre la membrana y la pantalla se encuentre entre $70 \mathrm{~cm}$ y $150 \mathrm{~cm}$ ). Posteriormente se debe conectar el celular con la App generadora de frecuencias al parlante, mientras que un segundo celular debe colocarse lo más próximo al parlante con la App para medir el nivel de sonido.

Fijar la distancia D, y fijar una frecuencia determinada (entre $200 \mathrm{~Hz}$ y $50 \mathrm{~Hz}$ ), variar el volumen del sonido y tomar las diferentes medida de la imagen en la amplitud mayor (L) que se forma y tomar los datos del celular que funciona como sonómetro y marcar los decibeles. Una vez obtenidas aproximadamente cinco variaciones de $\mathrm{L}$, cambiar la frecuencia y repetir la operación de toma de datos.

Se debe tener en cuenta que el ambiente donde se trabaje debe tener la mínima contaminación sonora posible para que no interfiera con el sonómetro. Obtenidos los datos de la Tabla 1, es posible graficar los decibeles en función de las amplitudes $\mathrm{L}$ para las distintas frecuencias (ver Gráfico 1).

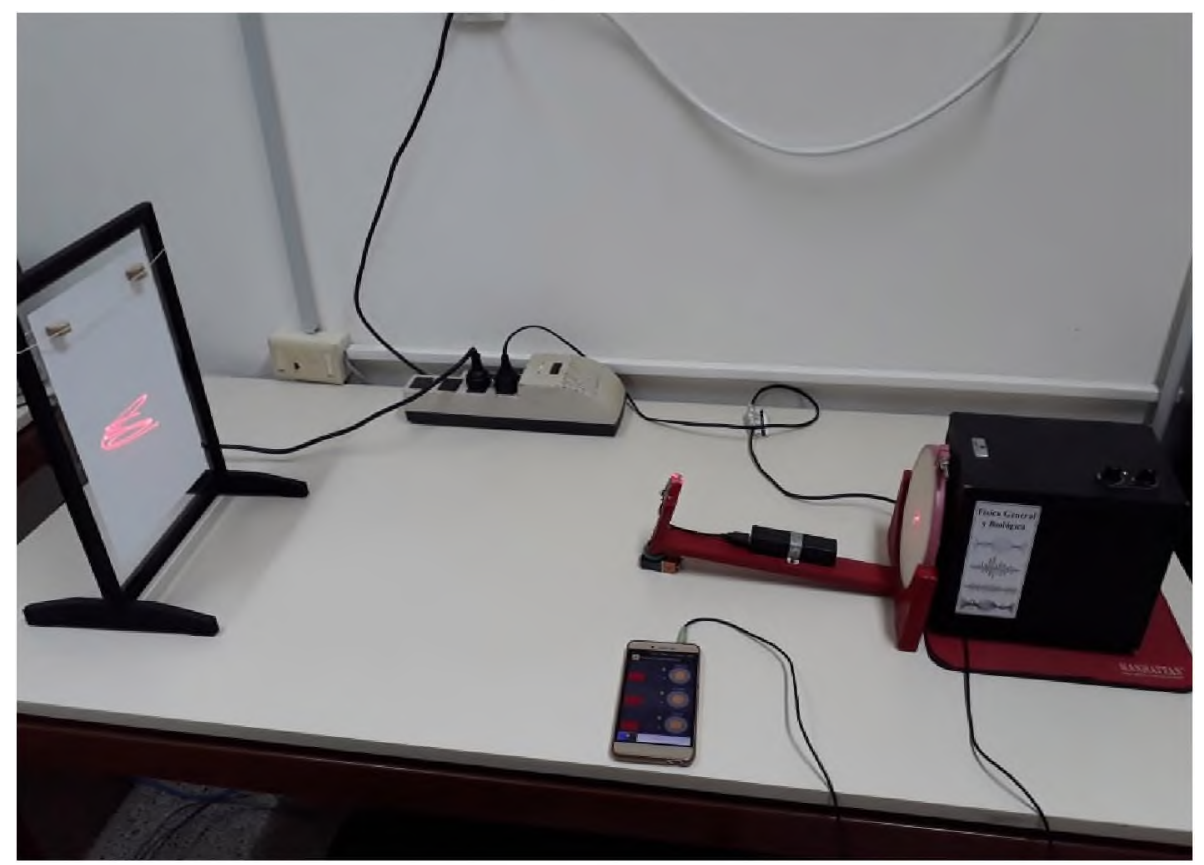

Figura 3: Fotografía del dispositivo funcionando junto con la APP Frequency Sound Generator

Como al incrementar el volumen del parlante se incrementa la amplitud de la figura de Lissajous y también los decibeles, es razonable pensar que ambas están relacionadas y que podriamos inferir la intensidad del sonido a partir de la amplitud de la figura para una distancia dada del espejo a la pantalla. 
La Tabla 1 muestra los valores de $\mathbf{L}$ tan bastante bien a una única curva que no $[\mathrm{cm}]$ y la sonoridad $\beta[\mathrm{dB}]$ para cuatro fre- depende de la distancia espejo-pantalla. De cuencias de la onda generada y cuatro dis- manera que la segunda parte de la hipótetancias, D, espejo-pantalla diferentes. Para sis planteada en el párrafo anterior deja de cada una de las frecuencias analizadas se tener relevancia en lo que sigue y nos conindican los datos obtenidos en el Gráfico 1. centramos en la relación entre la sonoridad Se puede observar que los puntos se ajus- $\beta$ y la amplitud $\mathbf{L}$.

Tabla 1: Medidas de la amplitud L en [cm] de la figura de Lissajous que se forma en la pantalla en función de la sonoridad $\beta$ en [dB] tomadas a distintas frecuencias de onda $[\mathrm{Hz}]$ y distancias espejo-pantalla $D[\mathrm{~cm}]$

\section{$50 \mathrm{~Hz}$}

\begin{tabular}{c|c|c|c|c|c|c|c}
\hline \multicolumn{2}{c|}{77} & \multicolumn{2}{c|}{90} & \multicolumn{2}{c|}{102} & \multicolumn{2}{c}{114} \\
\hline L & $\beta$ & L & $\beta$ & L & $\beta$ & L & $\beta$ \\
\hline 3,1 & 67,0 & 6,1 & 73,0 & 5,1 & 71,0 & 2,0 & 64,0 \\
\hline 4,9 & 71,0 & 7,8 & 75,0 & 8,2 & 74,0 & 4,9 & 71,0 \\
\hline 8,5 & 75,0 & 10,1 & 77,0 & 11,8 & 78,0 & 7,9 & 74,0 \\
\hline 12,2 & 79,0 & 15,0 & 80,0 & 15,3 & 80,0 & 11,1 & 77,0 \\
\hline 14,2 & 80,0 & 17,2 & 81,0 & 19,1 & 82,0 & 17,1 & 81,0 \\
\hline 17,2 & 81,0 & 19,7 & 82,0 & 22,2 & 84,0 & 24,7 & 85,0 \\
\hline
\end{tabular}

$90 \mathrm{~Hz}$

\begin{tabular}{c|c|c|c|c|c|c|c}
\hline 1,0 & 68,0 & 1,3 & 70,0 & 1,0 & 67,0 & 1,2 & 69,0 \\
\hline 1,4 & 71,0 & 1,8 & 74,0 & 1,6 & 73,0 & 1,7 & 73,0 \\
\hline 1,8 & 73,0 & 2,3 & 76,0 & 2,2 & 76,0 & 2,2 & 76,0 \\
\hline
\end{tabular}




\begin{tabular}{c|c|c|c|c|c|c|c}
1,0 & 15,0 & 2,3 & 10,0 & 2,2 & 10,0 & 2,2 & 10,0 \\
\hline 2,2 & 75,0 & 2,8 & 78,0 & 3,1 & 79,0 & 2,8 & 78,0 \\
\hline 3,1 & 79,0 & 3,5 & 81,0 & 4,0 & 82,0 & 3,1 & 79,0 \\
\hline 4,1 & 82,0 & 4,1 & 82,0 & 5,2 & 84,0 & 3,8 & 81,0 \\
\hline
\end{tabular}

$150 \mathrm{~Hz}$

\begin{tabular}{c|c|c|c|c|c|c|c}
\hline 6,3 & 76,0 & 4,1 & 72,0 & 8,1 & 77,0 & 3,0 & 70,0 \\
\hline 8,6 & 78,0 & 8,2 & 78,0 & 10,5 & 80,0 & 6,1 & 75,0 \\
\hline 11,8 & 80,0 & 11,1 & 80,0 & 14,2 & 83,0 & 11,4 & 80,0 \\
\hline 14,4 & 83,0 & 14,3 & 83,0 & 17,2 & 85,0 & 16,7 & 84,0 \\
\hline 17,4 & 85,0 & 16,8 & 84,0 & 20,8 & 86,0 & 20,6 & 86,0 \\
\hline 23,0 & 87,0 & 25,6 & 88,0 & 26,2 & 88,0 & 24,7 & 88,0 \\
\hline
\end{tabular}

$200 \mathrm{~Hz}$

\begin{tabular}{c|c|c|c|c|c|c|c}
\hline 1,2 & 64,0 & 1,4 & 64,0 & 1,7 & 65,0 & 1,4 & 64,0 \\
\hline 1,5 & 65,0 & 1,9 & 66,0 & 4,0 & 75,0 & 3,1 & 71,0 \\
\hline 2,0 & 67,0 & 2,5 & 70,0 & 5,4 & 78,0 & 3,5 & 73,0 \\
\hline 2,4 & 70,0 & 3,0 & 71,0 & 7,5 & 81,0 & 4,4 & 75,0 \\
\hline 3,3 & 71,0 & 4,5 & 76,0 & 8,4 & 82,0 & 4,9 & 77,0 \\
\hline 4,0 & 76,0 & 5,0 & 77,0 & 9,9 & 84,0 & 6,2 & 80,0 \\
\hline
\end{tabular}


Al graficar estos puntos encontramos la distancia $\mathbf{D}$ sino sólo de la frecuencia del que las curvas que forman no dependen de sonido, ver Gráfico 1.

Gráfico 1: Representación de los decibeles marcados por el sonómetro en función de las amplitudes $L$ en [cm] para las cuatro frecuencias analizadas $50 \mathrm{~Hz}, 90 \mathrm{~Hz}, 150 \mathrm{~Hz}$ y $200 \mathrm{~Hz}$.

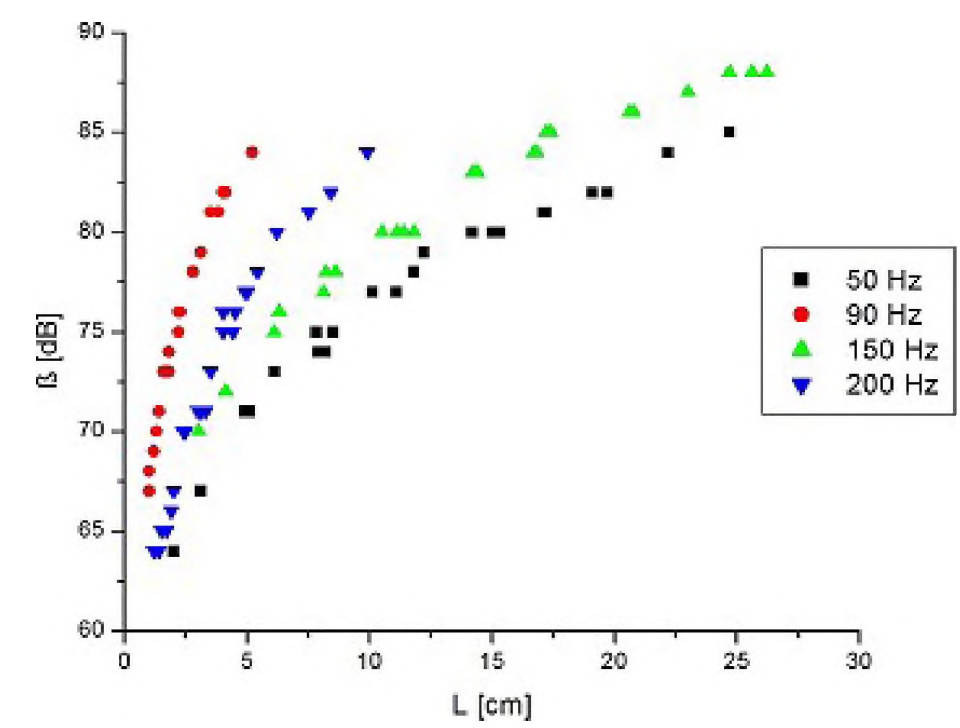

Como además ocurre que $\mathbf{D} \gg \mathbf{L}$, entre $\beta y$ el ángulo de apertura de la figura entonces $\mathrm{L}$ es aproximadamente igual al $\alpha \approx L /(2 D)$ La relación que encontramos arco que formaría la figura sobre una pan- entre la sonoridad y la amplitud de las imátalla esférica, es decir que la proporciona- genes de Lissajous es $\beta=a+b \cdot \operatorname{Ln}(\alpha)$ lidad entre $\beta y \mathbf{L}$ es idéntica a la que hay

Gráfico 2: Representación de los decibeles marcados por el sonómetro en función de las amplitudes

a)

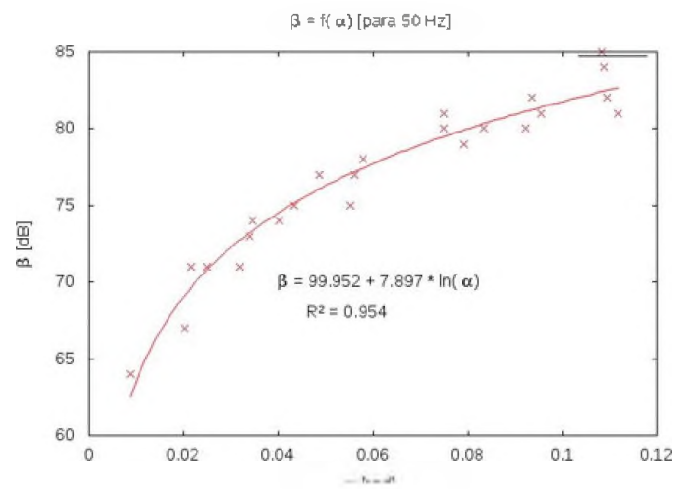

b)

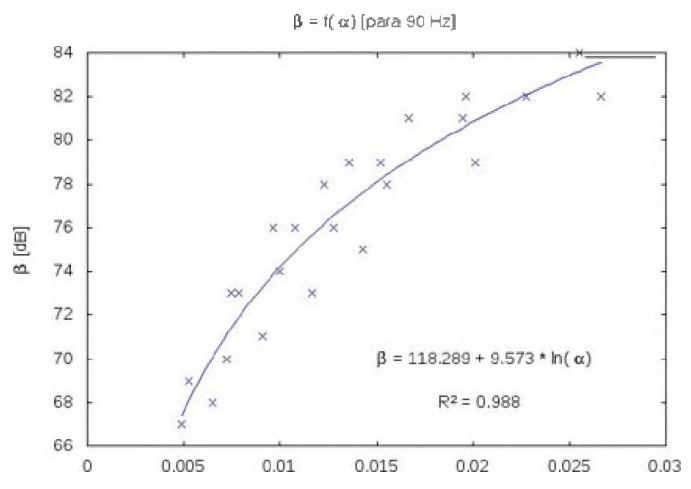


c)

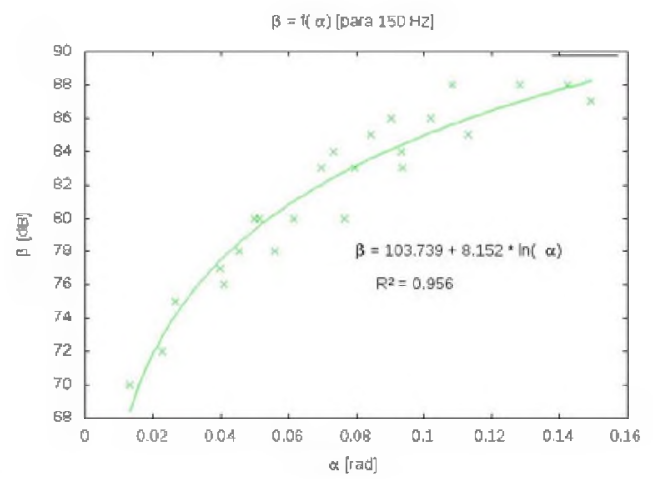

d)

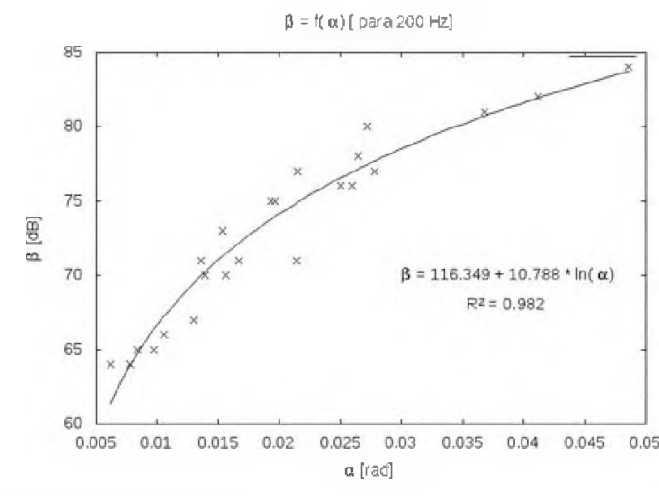

Por otro lado, para mostrar en el aula la formación de las figuras de Lissajous se puede simplemente seleccionar una radio o simplemente una canción en el celular conectado al parlante y observar como la membrana vibrante forma sucesivamente diferentes figuras en la pantalla. También, con la app generadora de frecuencias, se pueden formar algunas de las figuras de Lissajous que se muestran en la Figura 1 o alguna tabla más completa y proponer al alumno el reconocimiento de la misma identificando la relación que hay entre las frecuencias y fases de los movimientos armónicos simples.

\section{Conclusiones:}

Despertar el compromiso que debe tener el profesor contemporáneo en buscar nuevos espacios y modelos donde se pueda comprometer al estudiante en la importancia de la transversalidad del conocimiento y el adecuado uso de las herramientas com- putacionales para la solución de los problemas; es el desafío propuesto.

Un ejemplo claro y básico de la presentación de las figuras de Lissajous, puede darse a través de diferentes recursos informáticos como los que se describen a continuación. Estos van desde las elementales hojas de cálculo como EXCEL, pasando por lenguajes de programación como JAVA, o uno típico de animaciones como FLASH; hasta incluso emplear software científico como lo es, en este caso, MATLAB.

Sin embargo, con el diseño sencillo y de bajo costo del dispositivo presentado en este trabajo se espera generar motivación en los docentes para que promuevan procesos de formación en el aula en donde la transferencia del conocimiento sea un factor preponderante.

Además de mostrar las figuras de Lissajous se propuso la búsqueda de una función

$\beta=a+b \cdot \operatorname{Ln}(\alpha)$ que comparte varias herramientas y conocimientos transversales en la formación integral de los estudiantes. 
Así, consideramos a este tipo de desarrollos muy útil en el logro de aprendizaje significativo en el aula.

Es posible también pensar varias otras perspectivas en la forma de integrar el dispositivo "bardware" diseñado en este trabajo y algunas otras herramientas o conocimientos para llevarlo al aula de cursos básicos o avanzados.

\section{Agradecimientos:}

Este trabajo fue realizado gracias a la financiación recibida a través del Proyecto PI 17/F008 Res 0966/17 CS. de la Secretaría General de Ciencia y Técnica (SGCyT) de la Universidad Nacional del Nordeste (UNNE).

\section{Bibliografía:}

1. Cussó, F.; López, C.; Villar, R. (2004) Física de los procesos biológicos, $1^{\circ}$ edición, editorial Ariel. S.A. , Barcelona.

2. Cromer, A. (1981) Física para las ciencias de la vida. $2^{\circ}$ edición, editorial Reverte.

3. https://www.acusticaintegral.com/3296/ sonido-y-ruido/\#None. consultada el 24 de Octubre de 2018.

4. Cortés Osorio, J.A.; Montoya, F.; Chaves Osorio, J.A. (2008) Herramientas pedagógicas aplicadas en la enseñanza de las Figuras de Lissajous, Scientia et Technica Año XIV, $\mathrm{N}^{\circ}$ 40, Universidad Tecnológica de Pereira.

5. H. B. Miller, ed., Acoustical Measurements, Methods and Instrumentation, Benchmark Papers in Acoustics, Vol. 16. Stroudsburg:

Hutchinson Ross Publishing, 1982.

6. Física computacional aplicada en las figuras de Lissajous, Scientia et Technica Año XV, No 41, Mayo de 2009. Universidad Tecnológica de Pereira. ISSN 01221701 